\title{
Identifying Safe Zones for Planetary Satellite Orbiters
}

\author{
M.E. Paskowitz* and D.J. Scheeres ${ }^{\dagger}$ \\ University of Michigan, Ann Arbor, MI 48109
}

\begin{abstract}
This paper studies the dynamics of temporarily captured trajectories in the Hill 3-body problem, with application to orbits about Jupiter's satellite Europa. Using a model that includes the tidal force of Jupiter, the phase space of capture trajectories is numerically determined and trajectories are identified that don't impact with the planetary satellite or escape over long periods of time. These 'safe zones' in phase space are mapped out over different energy levels, identifying regions in phase space where an uncontrolled spacecraft would remain in orbit without impact or escape. The 'distances' of these safe trajectories from various frozen orbit trajectories are determined and the cost to transfer into these orbits is evaluated. Resulting from this analysis is the identification of robust capture trajectories and criteria on placing them into long-term stable orbits.
\end{abstract}

\section{Introduction}

In this paper, we study capture trajectories in the Hill 3-body problem. Capture trajectories are trajectories that begin in the exterior region of Hill's problem and enter the region to orbit the planetary satellite at least one time. Previous work has been done on these types of orbits. In particular Villac and Scheeres ${ }^{5}$ looked at escaping trajectories in the Hill 3-body problem, which are analogous to capture trajectories with time reversed. One particular feature of capture trajectories that we investigate is their lifetime. Unstabilized, these orbits can impact with the planetary satellite or exit the interior Hill region after vert short time spans. Koon et al. ${ }^{2}$ investigated orbits that travel between the interior and exterior Hill regions and showed that the amount of time a trajectory spends orbiting the planetary satellite is controlled by chaotic dynamics.

We develop a method which identifies sets of capture trajectories that do not impact or escape the planetary satellite for extended time periods. We call these 'safe trajectories' and the regions in which they lie 'safe zones'. These safe low-energy trajectories may be useful for the proposed NASA Jupiter Icy Moons Orbiter (JIMO) mission. The plan for this mission is to orbit three of Jupiter moons, Callisto, Ganymede and Europa. We focus our attention on Europa, however our results can be applied to the other moons as well. With the science goals of this mission being such that a low-altitude, high inclination stable orbit about Europa is desirable, we examine low-cost methods to transfer from a safe capture trajectory to a long-term stable orbit. In order to identify possible target long-term stable orbits, we turn to some previous work on orbits identified by using double averaging assumptions. There orbits are termed 'frozen orbits' and both circular and elliptic frozen orbits have been studied extensively. Broucke ${ }^{1}$ identified and studied the long term effects of both the circular and elliptic orbits. Scheeres, et al. ${ }^{4}$ studied circular frozen orbits with a specific application to orbits about Europa and showed that the unstable circular frozen orbits that exist at higher inclinations can be stabilized with small control maneuvers. Paskowitz and Scheeres ${ }^{3}$ studied elliptic frozen orbits with a particular application to orbits about Europa, and also investigated regions where the frozen orbit assumptions break down. Where these regions lie have strong consequences for our current study. Our analysis provides us with another method to determine where frozen orbit assumptions break down.

Along with these criteria for choosing appropriate target orbits, we also determine criteria on the safe capture trajectories that result in the lowest cost transfers. We discuss schemes that allow us to transfer to elliptic frozen orbits and circular frozen orbits and evaluate the costs associated with both schemes. We

*PhD Candidate, Department of Aerospace Engineering, mpaskowi@umich.edu

${ }^{\dagger}$ Associate Professor, Department of Aerospace Engineering, scheeres@umich.edu, Member AAS, Associate Fellow AIAA 
also provide specific examples of transfer maneuvers and the costs associated with them in order to identify possible ways to transfer to both elliptic frozen orbits and circular frozen orbits. The end result is a method that identifies trajectories enter into orbit about Europa on low-energy capture trajectories that do not impact or escape for at least one week, and from which it is possible to transfer to a more stable orbit with relatively low cost.

\section{Hill 3-Body Problem}

The physical system considered is that of a spacecraft orbiting a planetary satellite, which in turn is orbiting a planet. Figure 1 shows the geometry of this system. Only the first order perturbing effects of the planet's gravity is considered in this model, which is the Hill 3-body problem. This model is a limiting case of the circular restricted 3-body problem (CR3BP), and describes the dynamics of a massless particle which orbits two point masses rotating about each other in a circular orbit. The Hill 3-body problem gives a good description for the motion of a spacecraft in the vicinity of a planetary satellite, as validated in Scheeres, et al. $^{4}$

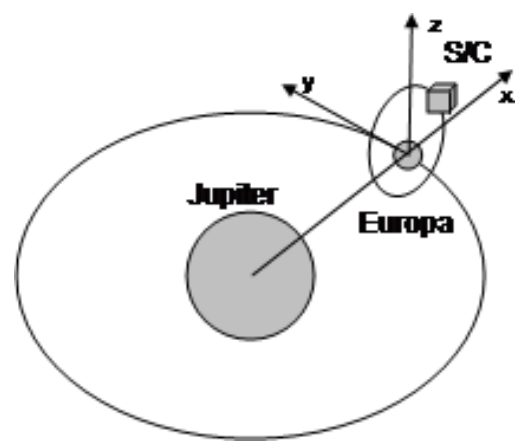

Figure 1. Geometry of the Europa orbiter model

The equations of motion for the Hill 3-body problem are as follows:

$$
\begin{aligned}
& \ddot{x}=2 N_{s} \dot{y}-\frac{\mu}{r^{3}} x+3 N_{s}^{2} x \\
& \ddot{y}=-2 N_{s} \dot{x}-\frac{\mu}{r^{3}} y \\
& \ddot{z}=\frac{\mu}{r^{3}} z-N_{s}^{2} z
\end{aligned}
$$

where $r=\sqrt{x^{2}+y^{2}+z^{2}}$ is the magnitude of the position vector, $N_{s}$ is the angular velocity of the planetary satellite about the planet, $\mu$ is the gravitational parameter of the planetary satellite, and their values for Europa are given in Table 1 . We assume that the planetary satellite is in a circular orbit about the primary.

\begin{tabular}{lcc}
\hline \hline Parameter & Symbol & Value \\
\hline Europa radius & $R$ & $1565 \mathrm{~km}$ \\
Europa orbital period & $T$ & $3.55 \mathrm{days}$ \\
Europa orbit rate & $N_{s}$ & $2.05 \times 10^{-5} \mathrm{rad} / \mathrm{s}$ \\
Europa gravitational parameter & $\mu$ & $3.201 \times 10^{3} \mathrm{~km}^{3} / \mathrm{s}^{2}$ \\
Normalizing unit length & $l=\left(\mu / N_{s}^{2}\right)^{1 / 3}$ & $1.968 \times 10^{4} \mathrm{~km}$ \\
Normalizing unit time & $\tau=1 / N_{s}$ & $4.878 \times 10^{4} \mathrm{~s}$ \\
\hline \hline
\end{tabular}

Table 1. Parameters of Europa

The equations of motion given in Eqs.(1)-(3) are in dimensional form. However, it is useful to nondimensionalize this model as computations can then be scaled to any physical system that is modeled by the Hill 
equations of motion. To nondimensionalize the equations of motion, take $l=\left(\mu / N_{s}^{2}\right)^{1 / 3}$ as the unit length and $\tau=1 / N_{s}$ as the unit time. The equations then take on the following form

$$
\begin{gathered}
\ddot{x}=2 \dot{y}-\frac{x}{r^{3}}+3 x \\
\ddot{y}=-2 \dot{x}-\frac{y}{r^{3}} \\
\ddot{z}=-\frac{z}{r^{3}}-z
\end{gathered}
$$

and have no free parameters.

Equilibrium solutions exist in the Hill 3-body problem, analogous to the $L_{1}$ and $L_{2}$ libration points in the CR3BP. There are two libration points that are symmetric about the origin with coordinates $x=$ $\pm\left(\mu / 3 N_{s}^{2}\right)^{1 / 3}, y=z=\dot{x}=\dot{y}=\dot{z}=0$ where the x-coordinate in nondimensional form is $x= \pm(1 / 3)^{1 / 3}=$ $\pm 0.693 \cdots$ (see Figure 2).

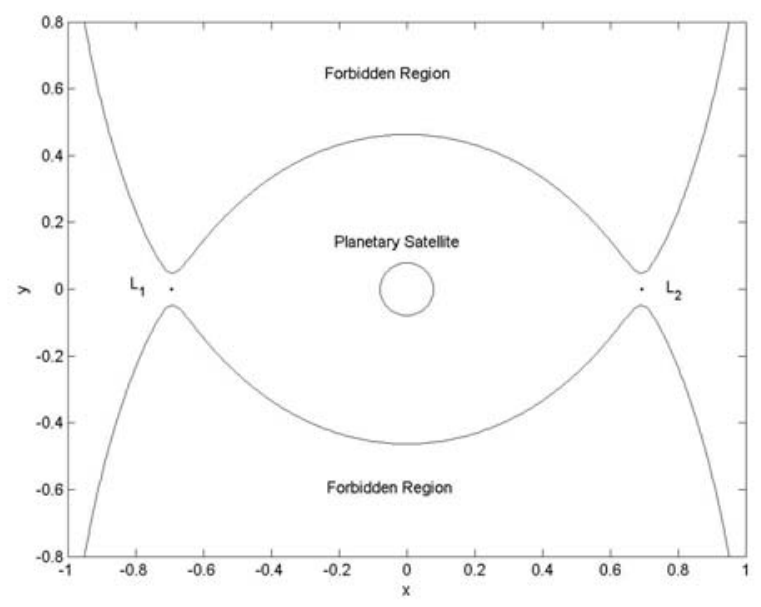

Figure 2. The regions of allowable motion and the libration points in the Hill 3-body problem at a Jacobi integral value of $J=-2.15$.

Equations 4-6 have an integral of motion, denoted as the Jacobi integral $J$ :

$$
J=\frac{1}{2} v^{2}-\frac{1}{r}-\frac{1}{2}\left(3 x^{2}-z^{2}\right)
$$

where $v=\sqrt{\dot{x}^{2}+\dot{y}^{2}+\dot{z}^{2}}$ is the speed of the particle in the rotating frame. The condition $v \geq 0$ in Eq.(7) places a restriction on the position of the particle for a given value of $J$. Setting $v=0$ defines the zero-velocity surface, which sets a physical boundary for the motion (see Figure 2).

For values of the Jacobi constant above a critical value, it is possible for both escaping and capture trajectories to exist in this problem. This critical value of $J$ defines the energy at which the zero-velocity surfaces open at $L_{1}$ and $L_{2}$ and is equal to

$$
J_{\text {crit }}=-\frac{1}{2} 9^{2 / 3}=-2.16337
$$

Since we are concerned with characterizing capture trajectories, we will look at trajectories with a Jacobi constant greater than $J_{\text {crit }}$.

\section{Periapsis Poincaré Maps}

A Poincaré map associates a continuous time dynamical system to a discrete time system. The use of a Poincaré map allows us to reduce the dimensionality of a system by at least one, and by two if there 
exists a first integral in the system, as is the case in the Hill 3-body problem. In general, Poincaré maps are defined such that the surface of section is a plane in position space, such as $x=0$. However, since all that is needed to define a Poincaré map are two surfaces of section that are transversal to the flow, we define them differently here, and use periapsis passages as our surface of sections, following Villac and Scheeres. ${ }^{5}$

\section{Definition of the Map}

Our Poincaré map relates capture trajectories that start near the libration point $L_{2}$ to the periapsis passages of these trajectories. Following Villac, ${ }^{6}$ we define the initial surface of section as the surface of a sphere with radius $\left(\frac{1}{3}\right)^{1 / 3}$ bounded by the zero-velocity surface. This surface then passes through the libration point region and extends as far as the zero-velocity surface of the Hill problem. The image surface is defined by the periapsis condition $\dot{r}=0$ and $\ddot{r}>0$. For the three-dimensional Hill 3-body problem, there are three degrees of freedom. The use of the Poincaré map allows us to restrict the problem to the surface of section, and hence reduce the problem by one dimension. As previously mentioned, there exists an integral of motion, $J$, in the problem, which allows us to reduce the problem by one additional dimension. The Poincaré map is then computed at a given value of the Jacobi integral $J$, (and so the map is a function of $J$ ), and is four-dimensional.

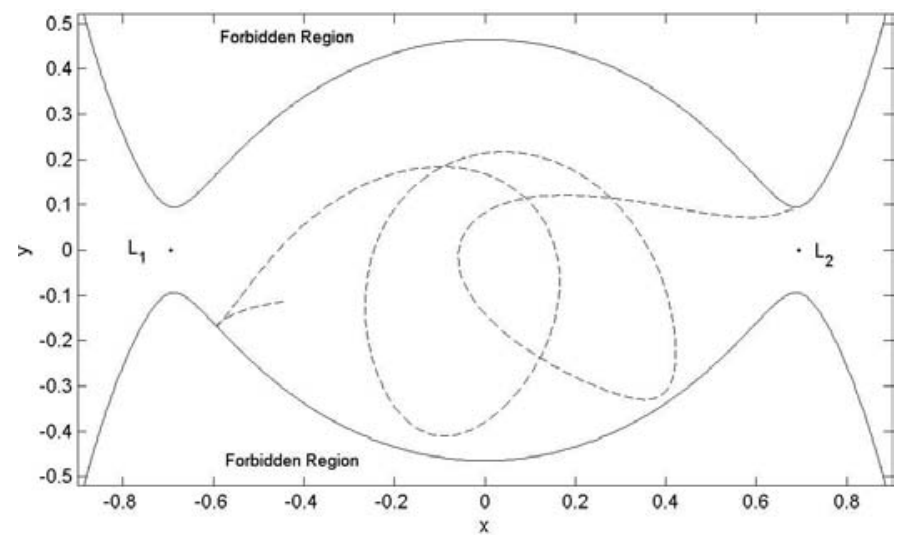

Figure 3. A planar capture trajectory with $\mathbf{J}=-2.15$ that originates near $L_{2}$ and orbits the planetary satellite.

Due to the symmetry between the $L_{1}$ and $L_{2}$ libration points, we only analyze capture trajectories that originate near $L_{2}$, however our results can be directly related to trajectories that originate near $L_{1}$ by the following transformation ${ }^{5}$

$$
(x, y, z, \dot{x}, \dot{y}, \dot{z}, t) \stackrel{G_{\mu}}{\longrightarrow}(-x,-y,-z,-\dot{x},-\dot{y},-\dot{z}, t)
$$

as the equations of motion remain unchanged. By a capture trajectory, we mean a trajectory that remains captured in the vicinity of the planetary satellite for a finite period of time. These trajectories enter the region of the planetary satellite in the vicinity of the libration point and orbit the planetary satellite at least once. Figure 3 shows an example of a capture trajectory.

In the three-dimensional case, the periapsis Poincare map is four dimensional and so we need four parameters to characterize it. For the initial surface of section, we use the $(\mathrm{x}, \mathrm{z})$ coordinates and two angles $(\delta, \phi)$ for the direction of the velocity vector. The $(x, z)$ coordinates are chosen randomly on the section of the surface of the sphere that falls within the allowable region. Since we are considering the surface of a sphere, the $y$ coordinate can be determined from the relationship $r=\sqrt{x^{2}+y^{2}+z^{2}}$, where $r=(1 / 3)^{1 / 3}$ and the sign for $y$ is chosen randomly. We then have the following initial conditions:

$$
\begin{array}{lr}
x_{0}=x & \dot{x}_{0}=v \cos \phi \cos \delta \\
y_{0}= \pm \sqrt{r^{2}-x^{2}-z^{2}} & \dot{y}_{0}=v \cos \phi \sin \delta \\
z_{0}=z & \dot{z}_{0}=v \sin \phi
\end{array}
$$


where the angles $(\phi, \delta) \in[\pi / 2,3 \pi / 2]$, and $v$ is determined from the Jacobi integral:

$$
v=\sqrt{2\left(J+\frac{1}{r}\right)+\left(3 x^{2}-z^{2}\right)}
$$

The parameters used to represent the image map are the periapsis position vector $(x, y, z)$ and the inclination.

With the initial conditions given by Eq.(10), an 8(7) order Runge-Kutta-Fehlberg integration routine is used to integrate the trajectories. We integrate the trajectories for multiple passages through the image map, meaning that they have multiple periapsis passages. We will study the phase space in terms of these multiple periapsis passages for various values of $J$.

\section{Poincaré Map Results}

As an initial study, we consider the two degree of freedom (planar) case of the Hill 3-body problem. In this model, motion is restricted to lie in the $\mathrm{x}-\mathrm{y}$ plane, and so the periapsis Poincaré map reduces to two dimensions. The initial surface, which consisted of the surface of a sphere in the three-dimensional case reduces here to an arc of circle. It is parameterized with the $x$ coordinate and an angle $\delta$ which defines the direction of the velocity vector. This is equivalent the the initial conditions defined by Eq.(10) with $z=\phi=0$. On the image map, we use the coordinates $(x, y)$ of the periapsis position vector.

Although the periapsis Poincaré map is computed in non-dimensional coordinates to allow for its application to many different physical systems, we will primarily consider Europa, one of Jupiter's moons. On many of the periapsis Poincare maps, the surface of Europa will be included to give some perspective to the plot. Although some of the periapsis passages of these trajectories lie beneath the surface of Europa, indicating that they impact with it, for the time being we will continue to include these trajectories in the analysis, since they are not necessarily impact trajectories when a different planetary satellite is considered. In a later section of this paper, we will deal with distinguishing between impacting and non-impacting trajectories.

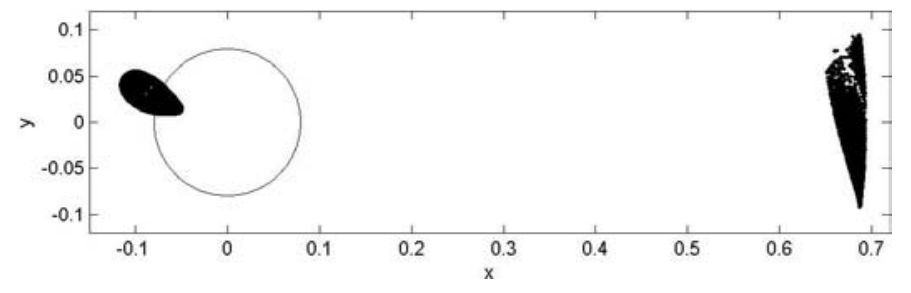

Figure 4. Periapsis Poincaré map for the planar case with $J=-2.15$ showing the first periapsis passage regions. The circle represents the surface of Europa.

We first consider the periapsis Poincaré map for the first periapsis passage of trajectories with $J=-2.15 .^{5}$ As shown in Figure 4 the first periapsis passages are divided into two disjoint regions. The first, located near the planetary satellite, corresponds to trajectories that immediately enter the region in the vicinity of the planetary satellite and have their first periapsis passage in that region. The second region, located near the $L_{2}$ libration point, corresponds to trajectories that do not immediately leave the vicinity of $L_{2}$ and have their first periapsis passage in that region. These can be associated with the periodic orbit that exists about $L_{2} .{ }^{6}$ Some of these trajectories will subsequently enter the region of the planetary satellite and some will escape from the system entirely. By our current definition of capture trajectory, there is no guarantee that the trajectory actually comes from outside the Hill region and not from inside the region itself. Even though our initial conditions stipulate that the trajectory must have an inward velocity vector it is possible that the trajectory does not originate from where we would like it to. To remedy this, we perform an additional integration. When necessary we integrate the trajectory backwards in time from its initial condition in the vicinity of the libration point for 4 time units which should give the trajectory enough time to exit the Hill region if that's what the trajectory corresponds to, and then only consider capture trajectories that lie beyond the boundary of the Hill region after those 4 times units. This guarantees that our capture trajectories actually do originate from outside the Hill region.

We now extend the periapsis Poincaré map to four periapsis passages. This means that we plot the first four periapsis passages of each trajectory for $J=-2.15$, as before. Each periapsis passage is assigned a 


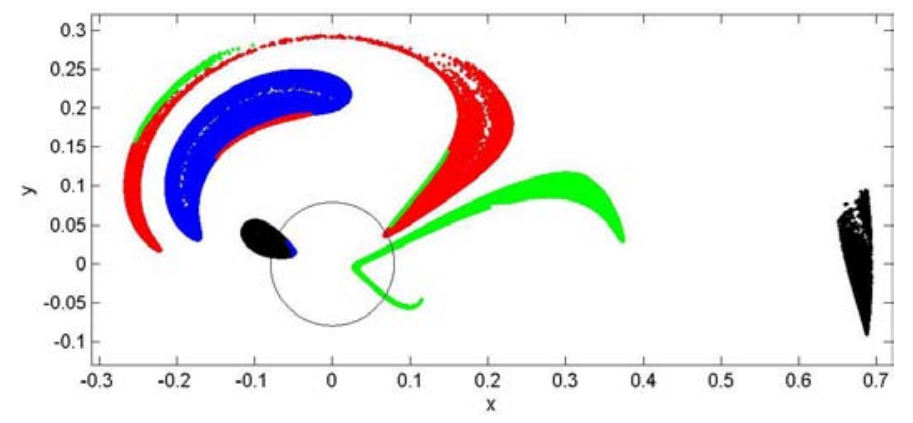

Figure 5. The first four periapsis passages for the planar case for capture trajectories with $J=-2.15$. The color-coding is as follows: black for 1st, blue for 2nd, red for 3rd and green for 4th periapsis passages. The circle represents the surface of Europa.

different color, as shown in Figure 5. We see that each subsequent periapsis passage region becomes more spread out. This occurs as we are not using symplectic coordinates on our map. We also note that some of the colors overlap (i.e, there is a small blue region inside the black region). These overlaps occur because, as previously mentioned, some of the trajectories do not immediately have a periapsis passage in the vicinity of the planetary satellite but instead have their first periapsis passage near the $L_{2}$ region. Therefore it is not until the second periapsis passage that these trajectories are near the planetary satellite, and so they have a different color. The dynamics of these trajectories, once they enter the region near the planetary satellite, are similar to the dynamics of the trajectories that enter that region immediately. It is also important to note that the gaps in some of the regions are not because trajectories cannot have periapsis passages there, but because these regions were generated numerically and, as such, only a finite number of trajectories can be considered.

From this point forward, we will ignore the region of first periapsis passages that occur in the vicinity of the $L_{2}$ libration point, and define the first periapsis passage of a particular trajectory to be its first periapsis passage which occurs in the vicinity of the planetary satellite.

To illustrate how the periapsis Poincaré maps depend on $J$, Figure 6 shows a series of Poincaré maps for increasing values of $J$. These Poincaré maps show only the first periapsis passage, since the trends shown by these regions will also apply to subsequent periapsis passages. We note that the regions become larger as $J$ increases, which is expected as the measure of the initial condition region increases with $J$.

It is much more difficult to visualize the periapsis Poincaré maps for the three-dimensional problem since the Poincaré map itself is four dimensional. In general, the trends we see in the two-dimensional case apply to the three-dimensional case, such as the periapsis passage regions becoming larger in position space as $J$ is increased as well as for subsequent periapsis passages. One way in which to visualize the Poincaré map for the three-dimensional case is to plot the periapsis passage's three-dimensional position vector, with an arrow indicating the direction of its velocity (which represents inclination). An example of this, for $J=-2.15$ is shown in Figure 7. Another way to visualize the three-dimensional case is by considering the inclination of the periapsis passage as a function of its radius. Figure 8 shows the inclination as a function of the radius for the first periapsis passage of trajectories with various values of $J$. We can see that as $J$ increases, the periapsis passages reach higher inclinations. Figure 9 shows the inclination as a function of the radius for multiple periapsis passages of trajectories with $J=-2.15$. In this case, the maximum obtainable inclination does not increase for subsequent periapsis passages, but the periapsis passages do occur over larger ranges of radii.

We can now introduce a more systematic description of these maps and regions. Define the set of trajectories that lie in the first periapsis passage region close to Europa as the set $S_{1}$,

$$
S_{1}=\{x \mid x \in \text { Initial Region }\}
$$

Then, under the flow dynamics of the Hill problem we can represent the Poincaré map as $\Phi$ where

$$
S_{n+1}=\Phi\left(S_{n}\right)=\Phi^{2}\left(S_{n-1}\right)=\cdots=\Phi^{n}\left(S_{1}\right)
$$

Thus, it is clear that any point in $S_{n+1}$ has a unique image in $S_{1}$. If we use the symmetry operator defined 

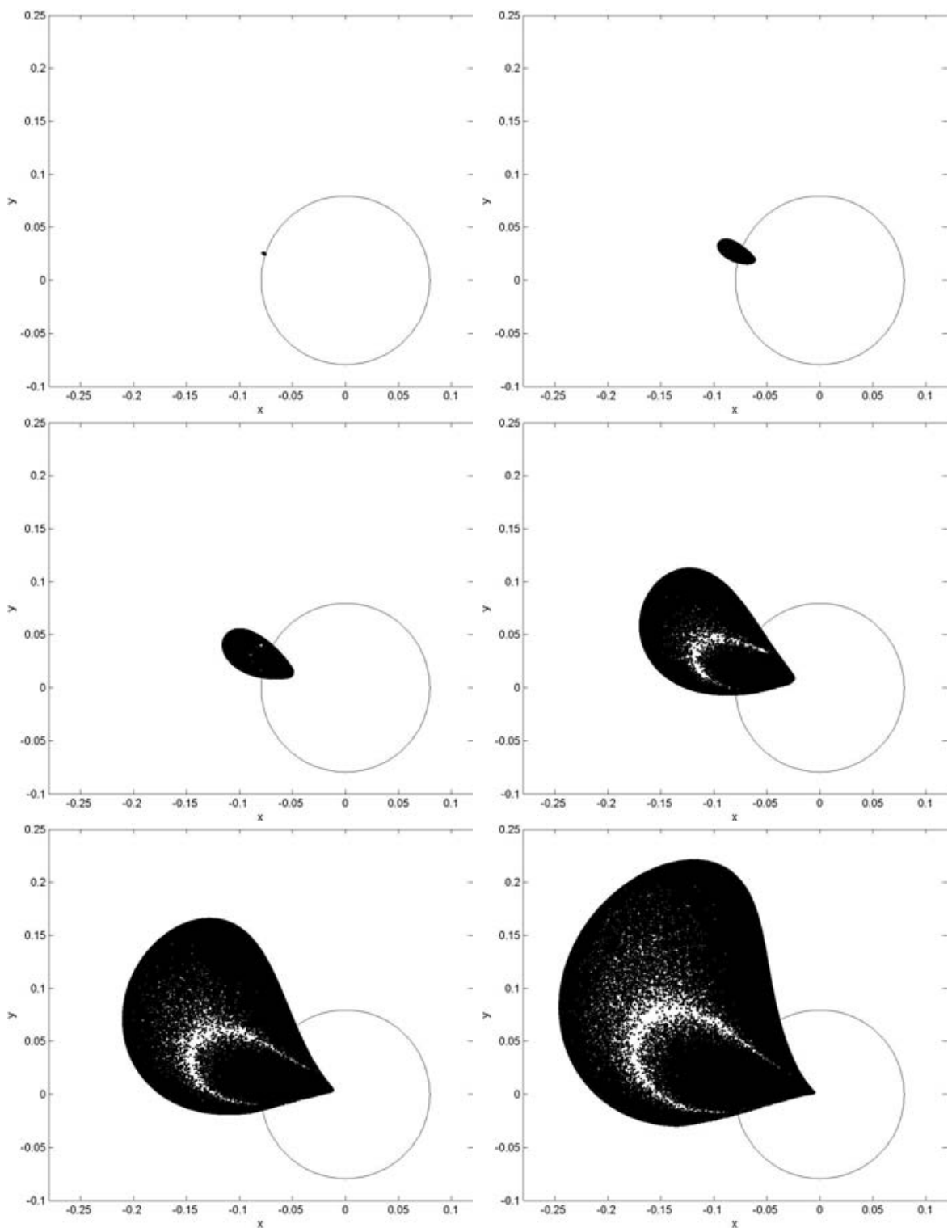

Figure 6. Series of Poincaré maps for various values of $J$. The circle in each plot denotes the surface of Europa. 


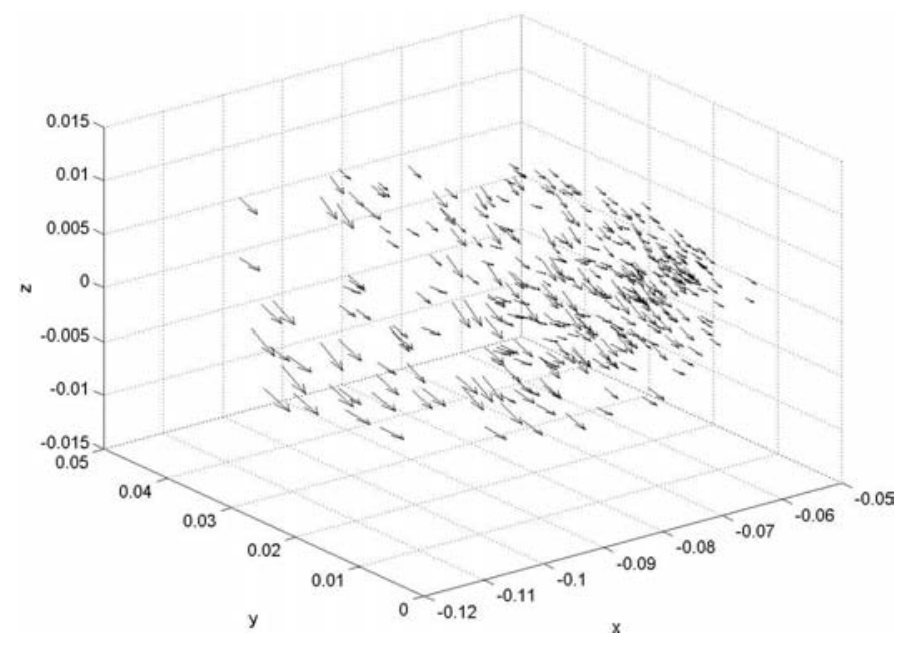

Figure 7. The first periapsis passage region for $3 \mathrm{~d}$ trajectories with $J=-2.15$. The location in position space of the periapsis passage is indicated by the base of the arrow, the velocity direction by the direction of the arrow and the magnitude of the velocity by the length of the arrow.

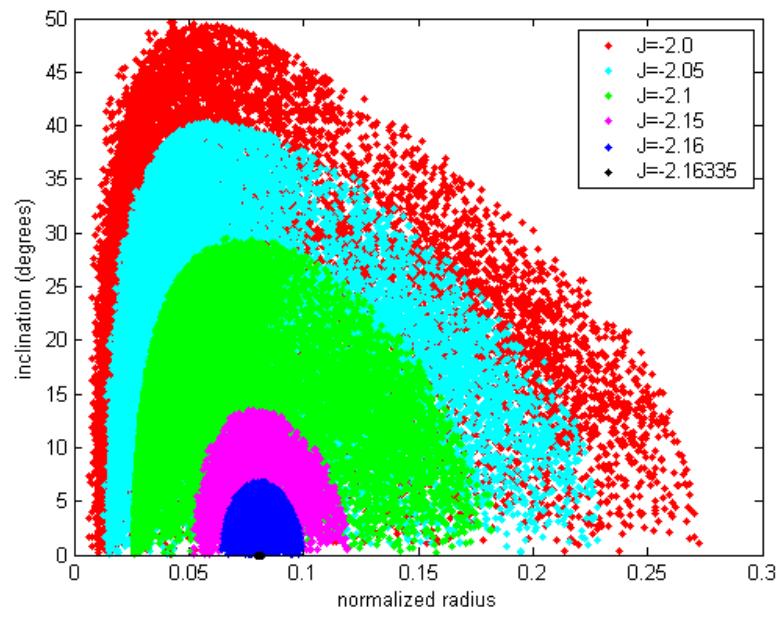

Figure 8. The inclination as a function of normalized radius for the first periapsis passage of trajectories with various values of $J$. As $J$ increases, the periapsis passages reach higher inclinations and occur over larger regions of radius. 


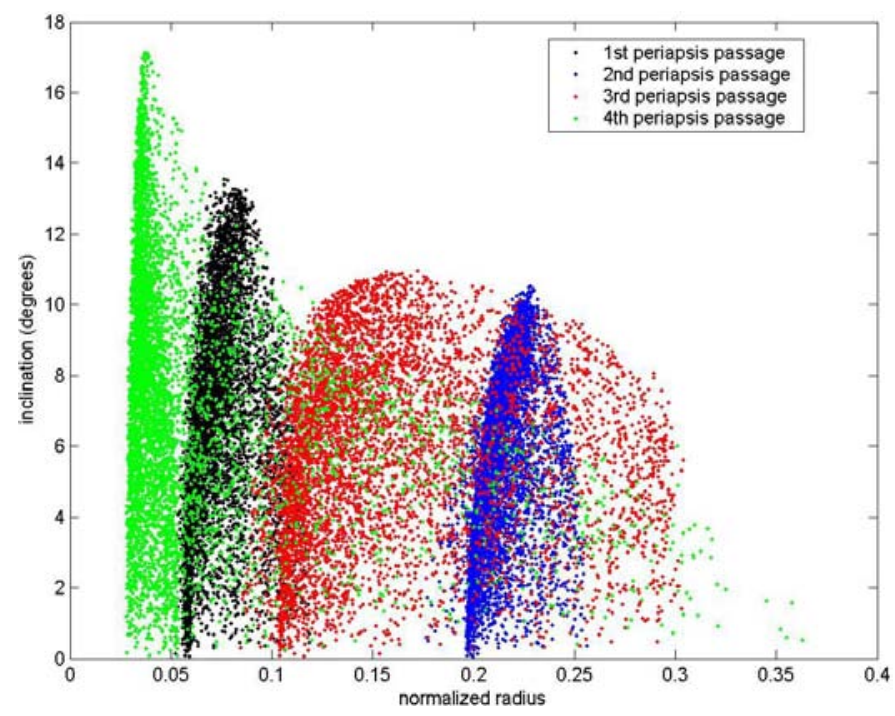

Figure 9. The inclination as a function of normalized radius for the first four periapsis passages of 3-dimensional capture trajectories with $J=-2.15$. The maximum obtainable inclination does not increase for subsequent periapsis passages, but does occur over a larger range of radii.

by Eq.(9), $G_{\mu}$, we can also associate the 'mirror image' trajectories as $S_{n}^{\prime}=G_{\mu}\left(S_{n}\right)$ defined by reflecting all coordinates and velocities about the origin.

\section{Symmetry Between Escape and Capture Trajectories}

Aside from the symmetry $G_{\mu}$ between $L_{1}$ and $L_{2}$ which was previously discussed, additional symmetries exist in this problem (and in the CR3BP). Two that we consider relate to the symmetry between escape and capture trajectories. If $(x, y, z, \dot{x}, \dot{y}, \dot{z}, t)$ is a solution of the equations of motion, then the trajectories obtained by applying the following transformations are also solutions: ${ }^{5}$

$$
\begin{gathered}
(x, y, z, \dot{x}, \dot{y}, \dot{z}, t) \stackrel{G_{t}}{\longrightarrow}(-x, y, x, \dot{x},-\dot{y},-\dot{z},-t) \\
(x, y, z, \dot{x}, \dot{y}, \dot{z}, t) \stackrel{G_{\mu} \circ G_{t}}{\longrightarrow}(x,-y, z,-\dot{x}, \dot{y},-\dot{z},-t)
\end{gathered}
$$

Thus if we have a capture trajectory, applying either of the above two symmetries will give us an escaping trajectory. By an escaping trajectory, we mean a trajectory that crosses the initial surface of sphere with an outward velocity, and therefore exits the Hill region. Figure 10 shows the symmetry between the capture and escape regions on the Poincare periapsis map for the first periapsis passage. This figure shows the first periapsis passage region for the capture trajectories, and hence for the escaping trajectories this corresponds to the periapsis passage immediately preceding escape (or transfer to the region in the vicinity of $L_{1,2}$.

This symmetry between capture and escape trajectories can be extended past the first periapsis passage. This allows us to determine when capture trajectories can escape from the Hill region. A capture trajectory escapes the Hill region when it passes through the initial surface of sphere with an outward velocity. However, a captured trajectory can not escape arbitrarily. We found that it will only escape when it passes through the 'first periapsis passage' escape region, which is the escaping region symmetric to the first periapsis passage capture region as shown on Figure 10. For this example $(J=-2.15)$, we found that the earliest that capture trajectories pass through this region is during their third periapsis passage, and so we can conclude that once a capture trajectory with $J=-2.15$ is found, it is guaranteed to remain captured for at least 3 periapsis passages. Such a trajectory must also lie in the pre-image of the escape region. Due to this, these transfer regions can be easily found by identifying regions where these symmetric regions intersect with each other. Figure 11 is a periapsis Poincaré map which shows the first four periapsis passages of capture trajectories as well as one set of their symmetric regions which correspond to escaping trajectories. We see that there is an overlap of these regions, which is the mechanism by which capture trajectories can subsequently escape. 


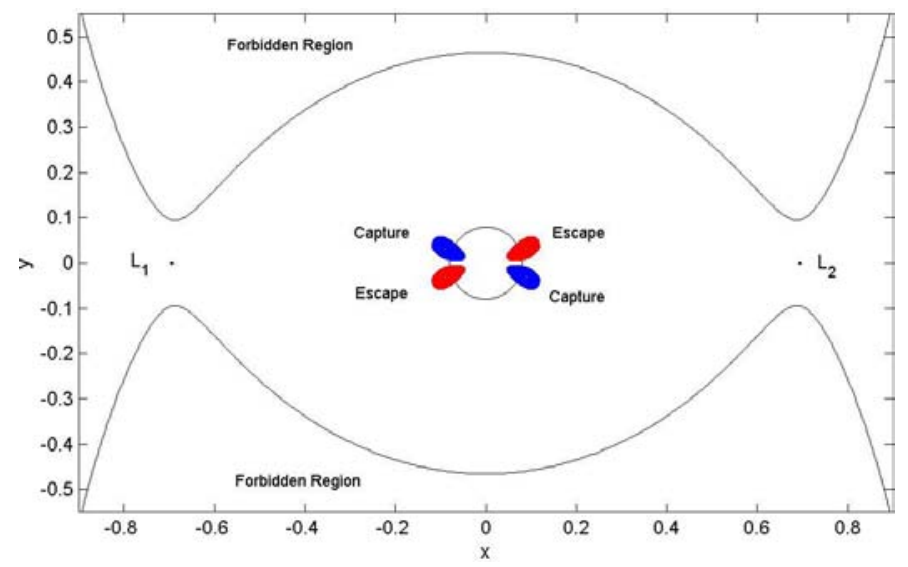

Figure 10. Poincaré maps showing the first periapsis passage regions for capture trajectories and the symmetric regions corresponding to escaping trajectories.

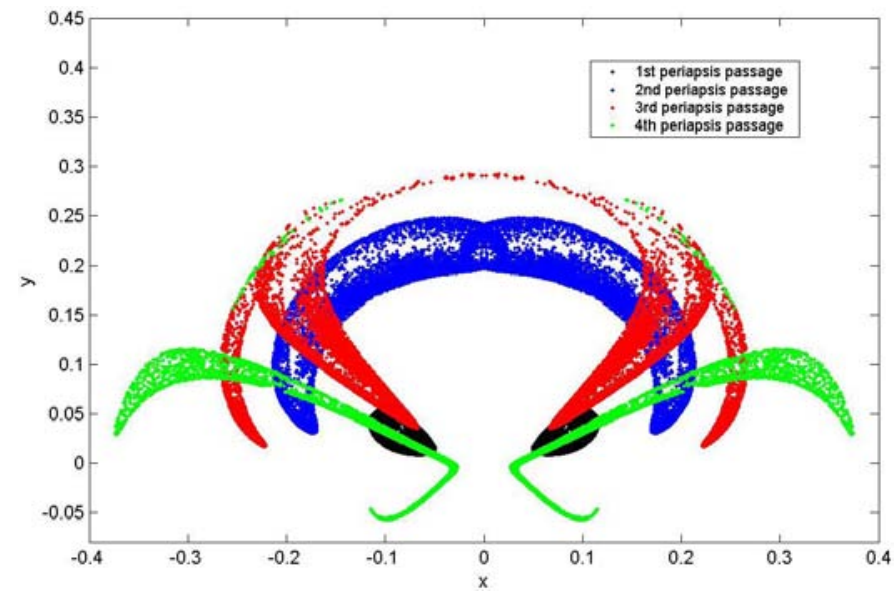

Figure 11. Periapsis Poincaré showing the first four periapsis passages of capture trajectories with $J=-2.15$ as well as the symmetric escape trajectory regions, reflected about the y-axis. The periapsis passages of capture trajectories are shown in red, and the periapsis passages of escape trajectories in blue, with the surface of Europa represented by the circle.

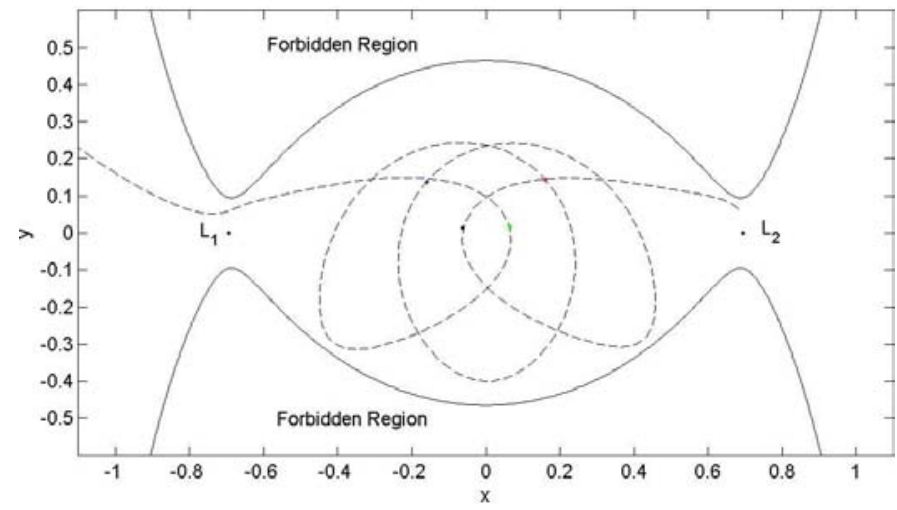

Figure 12. This is an example of a capture trajectory that escapes after four periapsis passages. The periapsis passage are indicated by the colored dots, where black is the first periapsis passage, blue the second, red the third and green the fourth. 
An example of this is shown in Figure 12. We can see that this trajectory has its third periapsis passage in the portion of the red region that overlaps with the blue symmetric region. We then know that its fourth periapsis passage will be in the portion of the green region that overlaps with the black symmetric region, which can be seen in Figure 11. Any capture trajectory which has a periapsis passage in the black symmetric region will not have any subsequent periapsis passages and will escape. Thus from the fact that this trajectory had its third periapsis passage in a region that overlaps with the blue symmetric escaping region we know that it will have a total of four periapsis passages and then escape. As seen in Figure 12, this trajectory does indeed escape from the Hill region after the fourth periapsis passages.

We can use the symbolic notation defined earlier to give a clear description of this result. First, we represent the time reversal symmetry by an operator $G_{t}$. From the property of this symmetry we have

$$
S_{-(n+1)}=G_{t}\left(S_{n+1}\right)=G_{t}\left(\Phi^{n}\left(S_{1}\right)\right)=\Phi^{-n} G_{t}\left(S_{1}\right)=\Phi^{-n}\left(S_{-1}\right)
$$

The condition for a trajectory $x_{1} \in S_{1}$ to escape is than that it eventually lies in $S_{-1}$ or $\Phi^{n}\left(x_{1}\right) \in S_{-1}$ for some $n$. If this occurs, it implies that $\Phi^{n}\left(S_{1}\right) \cap S_{-1} \neq 0$, or is not empty for some $n$, and that these particles escape in $n-(-1)=n+1$ iterations. In Figure 11 this region is represented by the overlap of the green points and the black points on the right side of the plot and we see that $\Phi^{3}\left(S_{1}\right) \cap S_{-1}$ is not empty and thus that they escape in 4 iterations from their initial location in $S_{1}$. Due to the uniqueness of solutions, we can also infer that the image of this set is invariant:

$$
\Phi\left(\Phi^{n}\left(S_{1}\right) \cap \Phi^{-m}\left(S_{-1}\right)\right)=\Phi^{n+1}\left(S_{1}\right) \cap \Phi^{-(m+1)}\left(S_{-1}\right)
$$

In Figure 10, we immediately note that for low values of $J$, we have $S_{1} \cap S_{-1}=S_{1} \cap G_{t}\left(S_{1}\right)=0$ and no immediate escapes occur. We also note, however, that when $J$ is large enough, the set $S_{1}$ crosses the $y=0$ axis, meaning that $S_{1} \cap G_{\mu}\left(G_{t}\left(S_{1}\right)\right) \neq 0$, where $G_{\mu}$ is the symmetry operator defined by Eq.(9), and thus that direct escapes can occur after one periapsis passage for higher $J$ values.

Although all of the figures in this section were computed for the planar case, the conclusions and the symbolic notation also apply to the three dimensional case where the sets are four-dimensional. In the three-dimensional case, analogous symmetric escaping regions exist, and we find that capture trajectories must have a minimum of three periapsis passages before escaping for low values of $J$. Figures computed for the planar case were used because this case is much easier to visualize graphically.

\section{Safe Zones}

We have previously discussed the possibility of capture trajectories eventually escaping from the Hill region, however we have not yet considered the trajectories that impact with the planetary satellite. In the Poincaré maps presented thus far all trajectories have been considered, even those whose periapsis passages clearly fall beneath the surface of the planetary satellite. To differentiate between trajectories that impact and trajectories that don't we introduce the term 'safe trajectory' to denote a trajectory that doesn't impact with the planetary satellite or escape from the Hill region for some specified period of time. The regions in which these trajectories lie will be denoted as 'safe zones'. In particular, we consider the first periapsis passage region $S_{1}$, and denote the set of all safe trajectories in the set the safe zone in $S_{1}$. We can then identify whether or not a trajectory is safe by where its first periapsis passage occurs. Although we define safe zones by the first periapsis passage of the trajectory, they could be defined by any of the periapsis passages. For example, we could have a safe zone defined by the second periapsis passage of the trajectories. In this case, we could say that any trajectory that had its second periapsis passage in that region is a safe trajectory. Since, as previously noted, the periapsis passage regions become distended for subsequent periapsis passages, the first periapsis passage region is the smallest, and hence is a logical choice for defining the safe zones.

We first consider safe zones in the planar case, and will continue to use Europa as our example. For this discussion, we consider that a trajectory is safe if it persists for a non-dimensional time period of $4 \pi$, which for Europa corresponds to 7.1 days. Figure 13 shows the safe zone in to the first periapsis passage of capture trajectories $\left(S_{1}\right)$ with $J=-2.15$. Capture trajectories with $J=-2.15$ with their first periapsis passage in the black region will not impact or escape for at least one week, the trajectories that lie in the red region will impact and the trajectories in the blue region will escape.

Figure 13 only shows the safe zone for one value of the Jacobi integral. We next consider the safe zones for various values of $J$. The results are shown in Figure 14 and are presented in a slightly different fashion, 


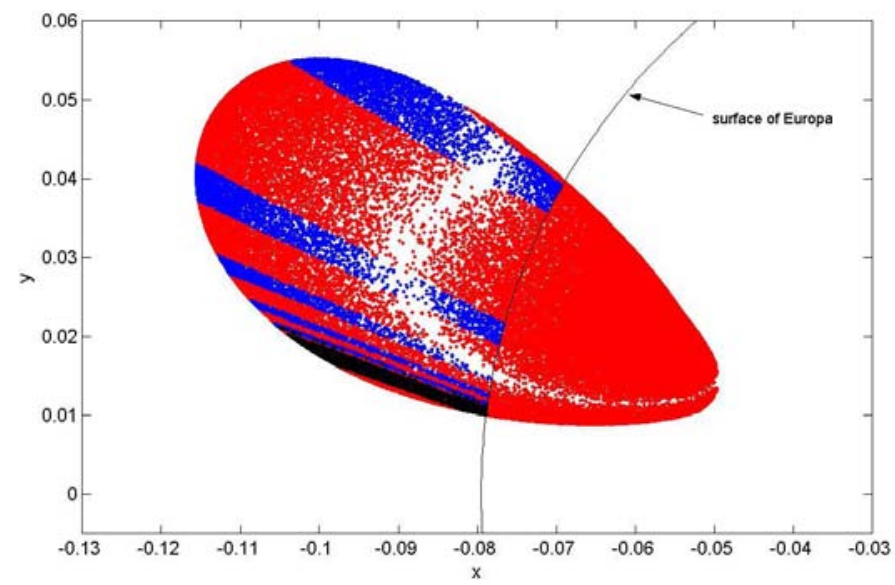

Figure 13. Poincaré map showing the safe zone, impact and escape regions. Trajectories were integrated for a period of time equivalent to one week, and their first periapsis passages are plotted. Trajectories that impact are shown in red, trajectories that escape in blue and trajectories that neither impact nor escape in black.

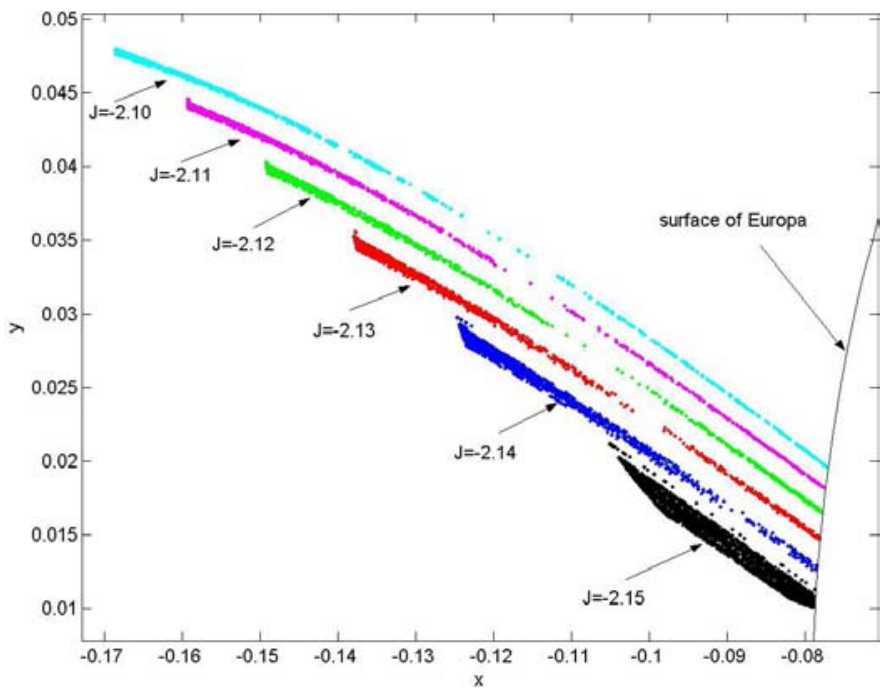

Figure 14. Poincaré maps of planar safe zones for various values of $J$ are plotted. The safe zones are shown in terms of their first periapsis passage. 
as only the safe zone is plotted, not the entire region of first periapsis passages. Once again, these maps are computed for $4 \pi$ time units and so do not impact or escape over that period of time. We see that as the Jacobi integral value increases, the safe zone regions become more distended and have a larger $y$ value.

By being able to characterize the safe zones in this way, we can conclude that if a trajectory has its first periapsis passage in the safe zone, if will be safe for at least one week. We can then, for a trajectory with a particular Jacobi integral value, determine where we wish its first periapsis passage to be. A trajectory that falls in this region is then potentially useful. If we wish to perform a maneuver to place it in a more stable trajectory but the maneuver fails, the spacecraft will remain safe for a period of time, hopefully long enough to perform another maneuver. Another motivation for characterizing these safe zones arises when considering low-thrust maneuvers. Since we know where in phase space the safe zones are located, low-thrust maneuvers can be designed such that the spacecraft travels through safe zones over the course of its maneuver, therefore guaranteeing that the spacecraft will not impact or escape.

The safe zones discussed above were computed for the planar case of the Hill 3-body problem. Analogous regions can be computed for the full three-dimensional case, however they will need to be studied in a different way. In this case, it is not only where the safe zone is located in position space that is important, but also its inclination. We could also characterize the three-dimensional safe zones in terms of the radius of the trajectory at its periapsis passage along with its inclination. However, it is not the visualization of the safe zones in the three dimensional problem that are important, but their existence. The method used to find safe zones for 3-dimensional trajectories is essentially the same as the method used in the planar case. We first identify capture trajectories for a particular value of $J$. Once we have found these capture trajectories, we determine whether or not they escape from the region or impact with the planetary satellite over a one week period for the Europa case. The capture trajectories that do not impact or escape during that time period are determined to be the safe trajectories.

\section{Transferring to a Long-Term Stable Orbit}

The preceding discussion defined a method to compute safe trajectories over a given period of time. For science operations, we wish to transfer the spacecraft into an orbit that is long-term stable. In the following we briefly review which orbits are stable over long time periods and determine the cost to transition into these orbits.

\section{Frozen Orbits}

Previous studies of the Hill 3-body problem have identified orbits that are stable over long time spans. These orbits can be found by analyzing a doubly averaged approximation to the Hill Problem. This approximation averages over both the mean anomaly of the orbit of the spacecraft about the planetary satellite and over one orbit of the planetary satellite about the planet. This allows us to obtain the secular equations of the orbital elements, where the change in the mean anomaly is not presented: ${ }^{4}$

$$
\begin{aligned}
\frac{d a}{d t} & =0 \\
\frac{d i}{d t} & =-\frac{15}{16} \frac{N_{s}^{2}}{n} \frac{e^{2}}{\sqrt{1-e^{2}}} \sin 2 i \sin 2 \omega \\
\frac{d e}{d t} & =\frac{15}{8} \frac{N_{s}^{2}}{n} e \sqrt{1-e^{2}} \sin ^{2} i \sin 2 \omega \\
\frac{d \omega}{d t} & =\frac{3}{8} \frac{N_{s}^{2}}{n} \frac{1}{\sqrt{1-e^{2}}}\left[5 \cos ^{2} i-1+5 \sin ^{2} i \cos 2 \omega+e^{2}(1-5 \cos 2 \omega)\right] \\
\frac{d \Omega}{d t} & =-\frac{3}{8} \frac{N_{s}^{2}}{n} \frac{\cos i}{\sqrt{1-e^{2}}}\left(2+3 e^{2}-5 e^{2} \cos 2 \omega\right)
\end{aligned}
$$

The numerical values of the parameters for Europa in the above equations are given in Table 1. Note that the equations do not depend on $\Omega$, and that $a$ is constant under this approximation, so we only need to consider the equations for $e, i$ and $\omega$. These three equations have equilibria, and it is these equilibria that will define the frozen orbits, orbits that have constant values of the orbital elements on average. As will be discussed, these frozen orbits correspond to viable trajectories, under some assumptions, in the unaveraged system. 
We first consider near-circular orbits. Previous studies have shown that near-circular frozen orbits exist for all values of inclination, and are stable for $i \leq 39.23^{\circ}$ and $i \geq 140.77^{\circ}$ and unstable for $39.23^{\circ}<i<140.77^{\circ} .4$ For the unstable orbits, the characteristic time is on the order of 14 days, where the orbital element value increases by an order of magnitude after $e \sim 2.718$ characteristic times. As long as the altitudes of these orbits are within a few hundred kilometers of the surface of Europa, these near-circular orbits will also exist in the unaveraged system. As the radius of the orbits get large, the tidal perturbations present in the system can cause the assumptions used to obtain the frozen orbits to become invalid. Once in this regime, most orbits are strongly unstable and are likely to impact or escape from the planetary satellite.

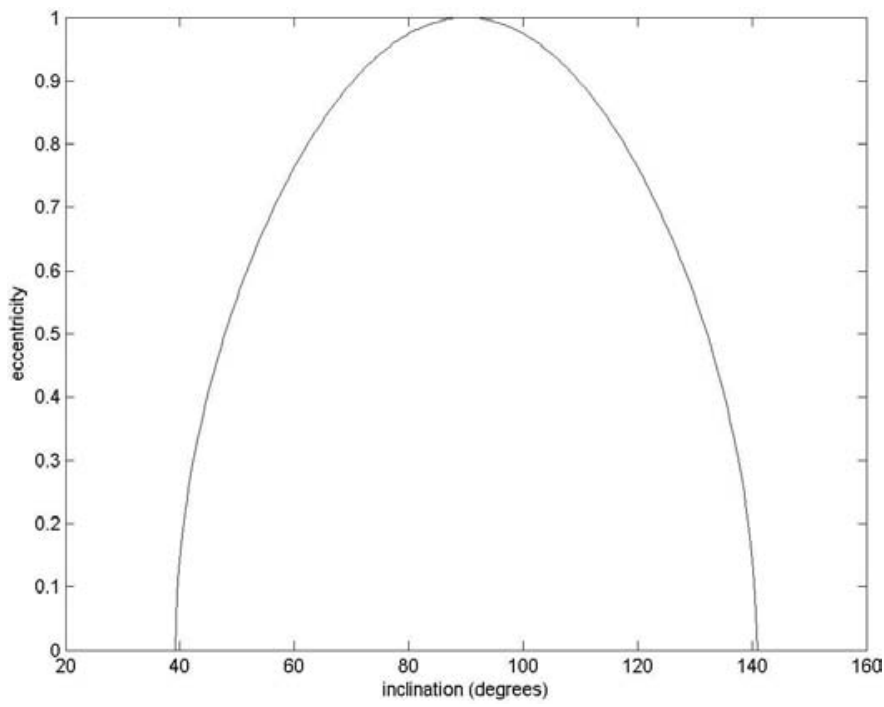

Figure 15. Relationship between inclination and eccentricity for elliptic frozen orbits.

Elliptic frozen orbits exist as well. By determining the equilibria of Eqs. 19-21, we find that elliptic frozen orbits exist for $\omega= \pm \pi / 2$ and $e=\sqrt{1-5 / 3 \cos ^{2} i}$, and these frozen orbits are stable whenever they exist. $^{3}$ Numerical simulations have shown that these frozen orbits also persist in the unaveraged system. However, there are limits on the applicability of the doubly averaged assumptions, and as the radius of these frozen orbits increase, the perturbations increase, and they are less likely to remain frozen and in orbit about the planetary satellite. Since, for the elliptic frozen orbits, the eccentricity and inclination are tied together, we can see that as the inclination becomes more polar, the eccentricity increases. Figure 15 shows the relationship between eccentricity and inclination for the elliptic frozen orbits. Therefore, as the orbit becomes more polar, the eccentricity increases and hence the apopasis radius increases, and so the perturbations on the orbit become stronger. We have previously found that the frozen orbit assumptions begin to break down and the frozen orbits cease to exist in the unaveraged system, for inclinations at $\sim 55-60^{\circ}$ for Europa. ${ }^{3}$

One way to visualize motion in the vicinity of elliptic frozen orbits is by using contour plots. There are two integrals of motion in the doubly averaged system, $\theta$, a term related to the angular momentum projected on the z-axis, and the doubly averaged potential $\tilde{R}:^{1}$

$$
\begin{gathered}
\theta=\left(1-e^{2}\right) \cos ^{2} i=\text { constant } \\
\tilde{R}=\frac{N_{s}^{2} a^{2}}{4}\left[1+\frac{3}{2} e^{2}-\frac{3}{2} \sin ^{2} i\left(1+\frac{3}{2} e^{2}-\frac{5}{2} e^{2} \cos 2 w\right)\right]
\end{gathered}
$$

The quantities $\tilde{R}=\tilde{R}(e, i, \omega)$ and $\theta=\theta(e, i)$ are integrals of motion, and thus are both constant. Based on this, we can define curves of ( $e$ vs. $\omega)$ and $(i$ vs. $\omega)$ that define where the motion occurs. These curves will be denoted as contour plots and define the path that the orbital elements will follow on average. For initial conditions that correspond to a frozen orbit, the contour plots have both circulating and librating regions, as shown in Figure 16, where the frozen orbit lies in the center of the libration region. Our goal is to perform a transfer maneuver such that the new orbit remains in the libration region. 

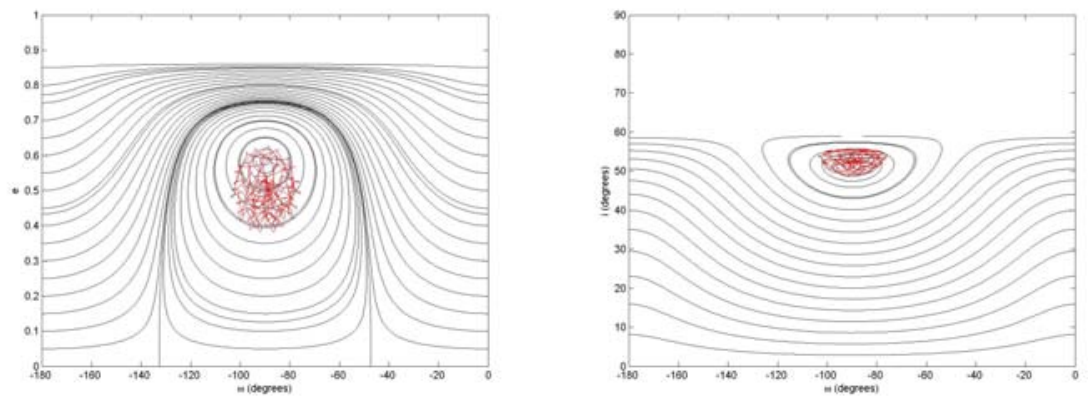

Figure 16. Examples of contour plots corresponding to the elliptic frozen orbit with $e=0.586$ and $i=51.14^{\circ}$.

\section{Using a Safe Capture Trajectory to Initiate a Transfer}

We have previously observed that it is desirable to perform maneuvers to transfer to a long-term stable orbit for capture trajectories in the safe zone. The elliptic and circular frozen orbits are such potential target orbits, and we first consider the elliptic frozen orbits. In order for this type of transfer to be affordable in terms of $\Delta v$, the safe trajectories and the frozen orbits must lie close to each other in phase space. So far we have been analyzing capture trajectories in terms of their periapsis passages. This becomes useful now, since an ideal location from which to initiate a transfer to a more stable orbit is at periapsis. Good science trajectories should have low periapsis altitudes, and so by performing the transfer at periapsis, we can ensure this. This is also the optimal place to make changes to the orbit energy.

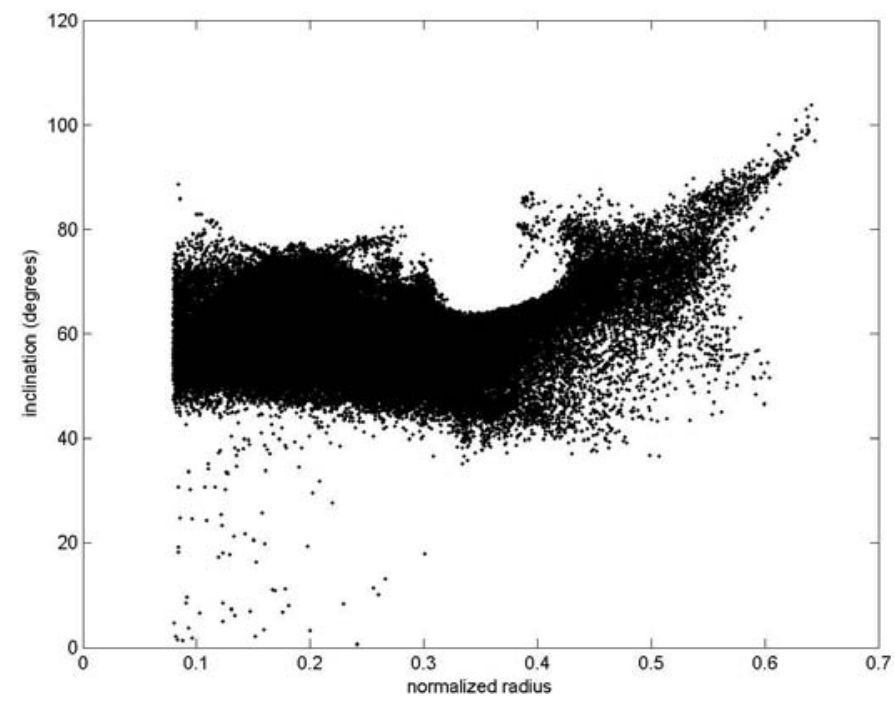

Figure 17. Inclination as a function of normalized radius for all of the periapsis passages of safe capture trajectories with $J=-1.70$. Note that the periapsis passages occur over a wide range of both radius and inclination.

When considering periapsis passages of capture trajectories from which to initiate a maneuver, we will consider all of the periapsis passages of these trajectories. Ideally, it would be better to use a periapsis passage that occurs near the beginning of the defined safe period, for obvious reasons. However, for now we consider any periapsis passage that occurs during the safe period. We first consider the safe zone for capture trajectories with $J=-1.70$ to see what range of radii they occur over. Figure 17 shows that the periapsis passages occur over a wide range of radii, all the way up to a normalized radius of almost 0.7 , which corresponds to $13,773 \mathrm{~km}$ (the radius of Europa is $1565 \mathrm{~km}$ ). We therefore see that we should restrict periapsis passages to those which have altitudes much closer to the surface. Figure 18 shows the same 


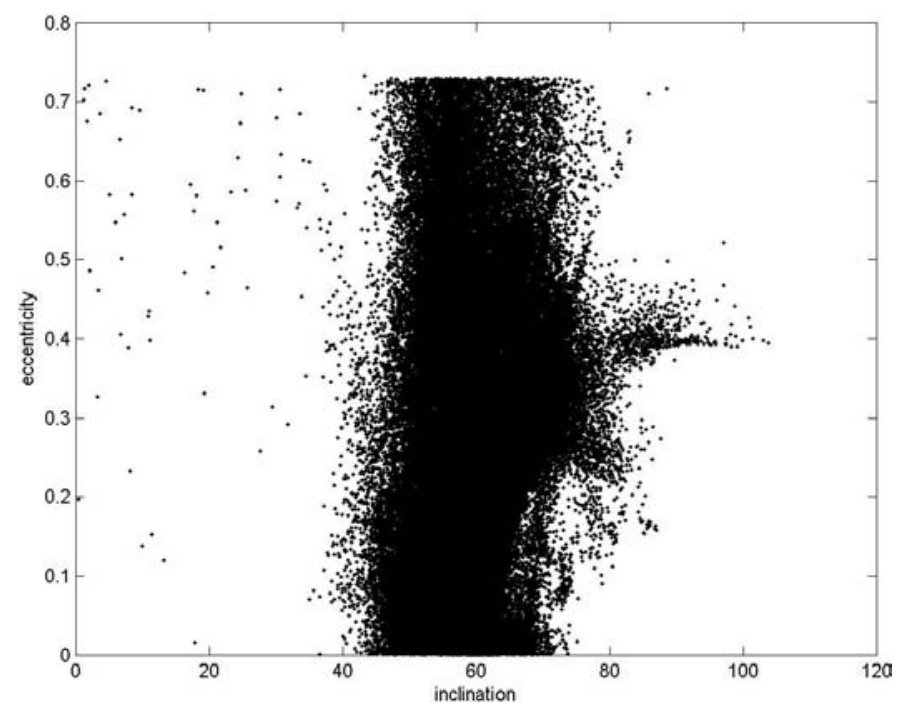

Figure 18. Eccentricity as a function of inclination for all of the periapsis passages of safe capture trajectories with $J=-1.70$. We can see that these periapsis passages occur over all possible eccentricities up to about 0.72 .

capture trajectories as Figure 17, but now showing eccentricity as a function of inclination. We see that the periapses of the safe capture trajectories occur over all eccentricities, up to about 0.72 .

\section{Transfer to Elliptic Frozen Orbits}

For transfers to elliptic frozen orbits, we restrict ourselves to periapsis passages of capture trajectories that are within $250 \mathrm{~km}$ of the surface of Europa. Figure 19 shows the eccentricity as a function of inclination for periapsis passages of safe capture trajectories that satisfy this condition for different values of $J$. As $J$ increases, the periapsis passages reach higher inclinations. We also see that restricting the periapsis passages to fall within $250 \mathrm{~km}$ of the surface of Europa causes a large reduction in the range of eccentricities over which they occur. For example, if we compare the part of Figure 19 that corresponds to the capture trajectories for $J=-1.70$ to Figure 18, we see that when the radius of periapsis is not restricted, the periapsis passages had eccentricities over the range of $e=0 \rightarrow 0.72$ and when the radius of periapsis is restricted, the eccentricity only ranges over about $e=0.68 \rightarrow 0.72$. Therefore we conclude that the lower eccentricity periapsis passages of the safe capture trajectories occur at larger radii and hence are farther from the surface of Europa.

Recall that the elliptic frozen orbits have orbit elements $\omega= \pm \pi / 2$, and $e=\sqrt{1-5 / 3 \cos ^{2} i}$. Since we wish to transfer from a safe capture trajectory to a frozen orbit, we must determine what characteristics the periapsis passages of the capture trajectories must have in order to perform this maneuver. The first thing we do is only consider periapsis passages of capture trajectories that have an argument of periapsis close to the frozen orbit value. Since there are oscillations in the frozen orbits in the unaveraged system, we can relax the condition $\omega= \pm 90^{\circ}$ somewhat and consider periapsis passages of safe capture trajectories that fall within at least 5 degrees of this condition. Then, if the periapsis passage of the safe capture trajectory has an inclination for which an elliptic frozen orbit exists, a maneuver can be performed to change the eccentricity so it corresponds with the inclination based on the frozen orbit relation, and the spacecraft would then be in an elliptic frozen orbit.

Figure 20 shows the set of periapsis passages of safe capture trajectories with $J=-1.60$ that lie within $250 \mathrm{~km}$ of the surface of Europa and have an argument of periapsis within 5 degrees of $\pm 90^{\circ}$. A line denoting where the elliptic frozen orbits lie is shown, and so we see the change in eccentricity that would be required to transfer from a capture trajectory to a frozen orbit. We see that the frozen orbit line actually passes through the safe zone. This implies that the assumptions of the doubly averaged system are not valid at and beneath the frozen orbit line and hence provides an indication of the eccentricity and inclination at which the frozen orbit assumptions break down. This should be clear, as the trajectory originally came from outside the Hill sphere. When considering safe capture trajectories from which to initiate a transfer, we do not consider periapsis passages that fall underneath the frozen orbit line, since this is beyond the point at 


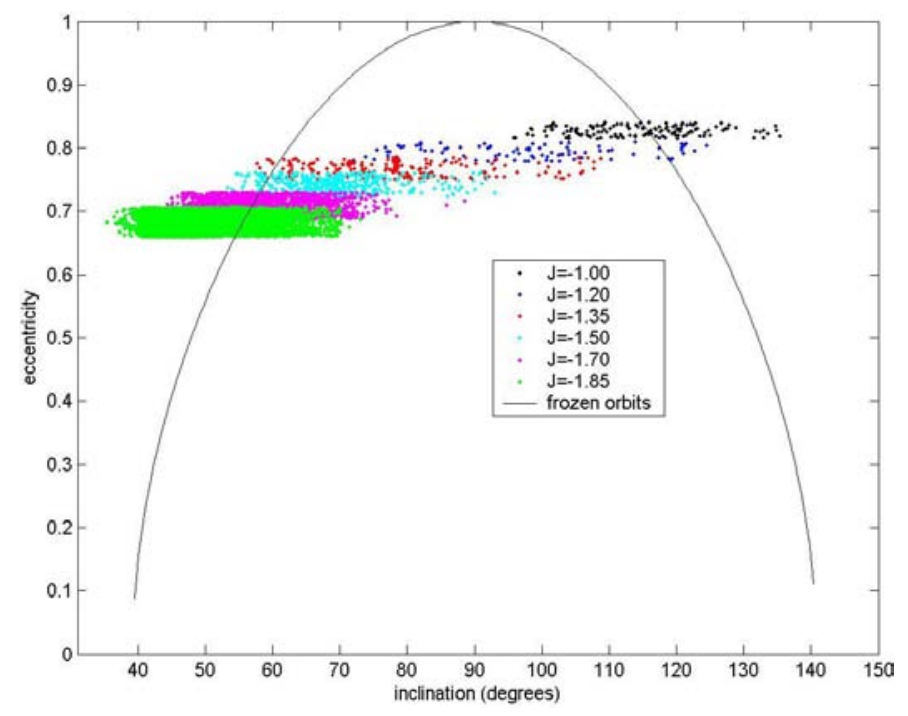

Figure 19. The periapsis passages of capture trajectories with different values of $J$ that could be used to initiate a transfer to an elliptic frozen orbit. The periapsis passages were restricted to have an altitude within $250 \mathrm{~km}$ of the surface of Europa.

which the averaging assumptions break down.

Figure 21 shows the cost, in meters per second, to transfer from the safe capture trajectory to an elliptic frozen orbit. The periapsis passages of safe capture trajectories used to initiate the transfer are those shown in Figure 20. The region in the figure that is denoted 'not valid' refers to the region where the frozen orbits have broken down. The region denoted 'valid' refers to the fact that these transfers are practical since the frozen orbits exist. The gray region is present since we do not have an absolute boundary on where the frozen orbits cease to exist, and so some transfers in the gray region may be valid while others are not.

To prove that our scheme to transfer to an elliptic frozen orbit works, we computed the transfer for one of the periapsis passages of the safe capture trajectories and then continued the integration to show that we do indeed transfer to a stable elliptic frozen orbit. Starting from the safe capture trajectories with $J=-1.60$, as shown in Figure 20, we choose the periapsis passage that has the following parameters: $e=0.718$, $\omega=-93.66^{\circ}, i=51.14^{\circ}$, and a radius of periapsis $166 \mathrm{~km}$ above the surface of Europa. The eccentricity that corresponds to a frozen orbit at this inclination is 0.586 . The cost of performing this maneuver is 69 $\mathrm{m} / \mathrm{s}$, and the resulting trajectory, from the point where the capture trajectory enters the Hill region until 56 days after the transfer at which point the frozen orbit is still stable, is shown in Figure 22. To show that the new orbit is an elliptic frozen orbit, we plot the trajectory on contour plots of eccentricity as a function of argument of periapsis and inclination as a function of argument of periapsis. Figure 23 shows that the new orbit (plotted in red) does stay inside the libration region of the contour plot, and so we have successfully transferred from a safe capture trajectory to an elliptic frozen orbit.

\section{Transfers to Circular Frozen Orbits}

We currently consider two approaches to transfer to a circular frozen orbit, two-impulse and one-impulse maneuvers. The two impulse scheme involves performing a maneuver at the periapsis passage of the capture trajectory that allows us to use the dynamics of elliptic frozen orbits to achieve a lower eccentricity (which in turn increases the inclination). Following that, a second maneuver is performed at the much lower eccentricity to circularize the orbit. The one-impulse maneuver involves circularizing directly from the periapsis passage of the capture trajectory.

We first consider the two-impulse approach. Previously, in order to transfer to an elliptic frozen orbit, we chose a maneuver to achieve the frozen orbit eccentricity. By examining the left plot in Figure 16 we see that if we choose a slightly higher eccentricity, we can achieve much larger variations in eccentricity, to the point where the eccentricity occasionally gets very close to 0 . Figure 24 shows this phenomenon, where instead of performing a maneuver to change the eccentricity to 0.586 as above, we change the eccentricity to 0.687. 


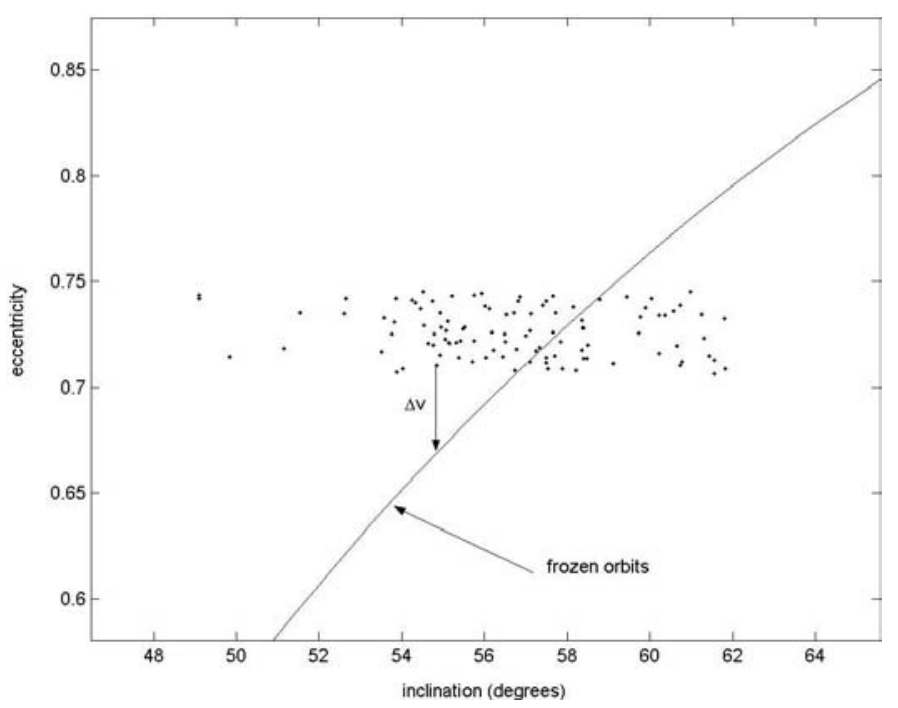

Figure 20. The set of periapsis passages of safe capture trajectories with $J=-1.60$ that lie within $250 \mathrm{~km}$ of the surface of Europa and have an argument of periapsis within 5 degrees of $\pm 90^{\circ}$. The solid line denotes where the elliptic frozen orbits lie and the change in eccentricity required to transfer from a capture trajectory to an elliptic frozen orbit is shown as $\Delta v$.

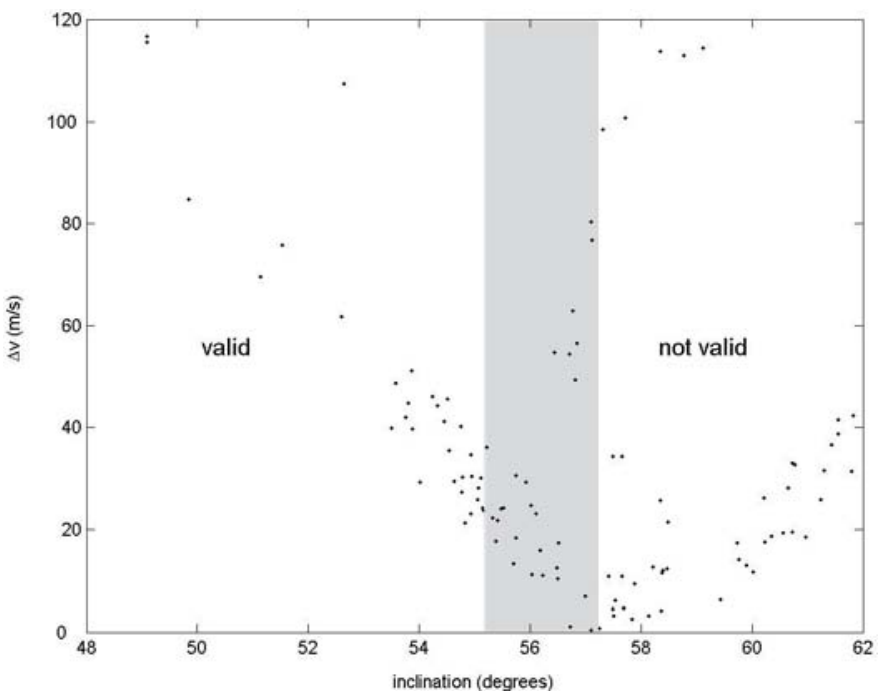

Figure 21. The cost in meters per second to transfer from the safe capture trajectory to the corresponding elliptic frozen orbits. The transfers are divided into three categories: those that are not valid due to large perturbations of the frozen orbit, those that are valid since the frozen orbits exist in the unaveraged system, and the grey region where some frozen orbits are valid and some are not. 


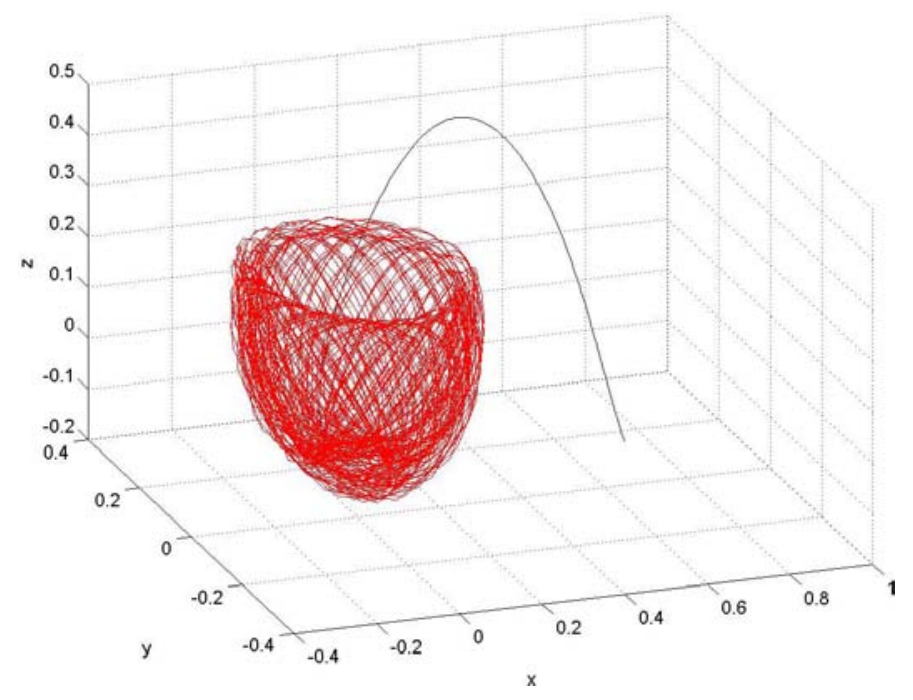

Figure 22. An example of a transfer from a safe capture trajectory with $J=-1.60$ to an elliptic frozen orbit.
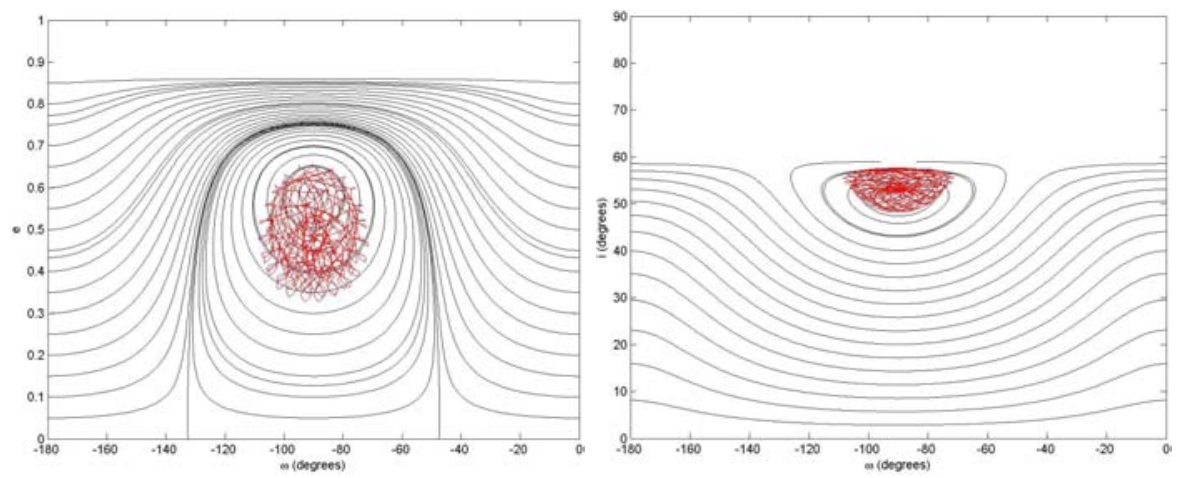

Figure 23. Contour plots for eccentricity and inclination as function of $\omega$ are plotted with the orbit obtained after the transfer maneuver. 
We use the same periapsis passage as in the elliptic frozen orbit transfer example (i.e, $e=0.718, i=51.14^{\circ}$, $\omega=-93.66^{\circ}$, altitude $\left.=166 \mathrm{~km}\right)$. The cost of this maneuver is $38.6 \mathrm{~m} / \mathrm{s}$. The next step in this approach is to circularize the orbit. Depending on the altitude of the orbit after it's circularized, some control maneuvers may be necessary to keep the orbit from drifting too much. The frequency and size of these maneuvers will depend on how much of a drift we can tolerate and how quickly the orbit is drifting (depending on its altitude).

The circularization maneuver will be performed at a periapsis passage of the orbit shown in Figure 24 . In order to understand how the radius, eccentricity and inclination of the orbit vary and where the periapsis passages occur, we refer to Figure 25 which shows the radius, eccentricity and inclination as functions of time. The periapsis passages are indicated with a red ' $x$ ' and the solid blue line in the top plot represents the surface of Europa. We see that a lower eccentricity is correlated to a higher radius and higher inclination. Higher inclinations are more desirable, however the higher radius associated with the higher inclination will mean an initially large circular orbit. To compare these issues, two examples will be provided: one where the circularization maneuver is performed from the minimum eccentricity of the orbit shown in Figure 24 and one where the circularization is performed from a higher eccentricity. In both cases, the radius of the orbit will be relatively large and some control maneuvers will be necessary to maintain this orbit. Table 2 shows the cost of achieving both of these circular orbits as well as their average radius and initial inclination. Figure 26 shows their characteristics.

\begin{tabular}{lcc}
\hline \hline & Example 1 & Example 2 \\
\hline From capture trajectory to near-frozen elliptic orbit & $38.6 \mathrm{~m} / \mathrm{s}$ & $38.6 \mathrm{~m} / \mathrm{s}$ \\
Circularization & $10.3 \mathrm{~m} / \mathrm{s}$ & $230.3 \mathrm{~m} / \mathrm{s}$ \\
Total Cost & $48.9 \mathrm{~m} / \mathrm{s}$ & $268.9 \mathrm{~m} / \mathrm{s}$ \\
Average Radius & $5300 \mathrm{~km}$ & $2875 \mathrm{~km}$ \\
Initial Inclination & $61.6^{\circ}$ & $57.9^{\circ}$ \\
\hline \hline
\end{tabular}

Table 2. Comparison of the cost to transfer from the periapsis passage of a capture trajectory to a circular frozen orbit. Example 1 corresponds to the circularization taking place at the minimum eccentricity of the near-frozen elliptic orbit and example 2 corresponds to using a higher eccentricity from which to circularize from.
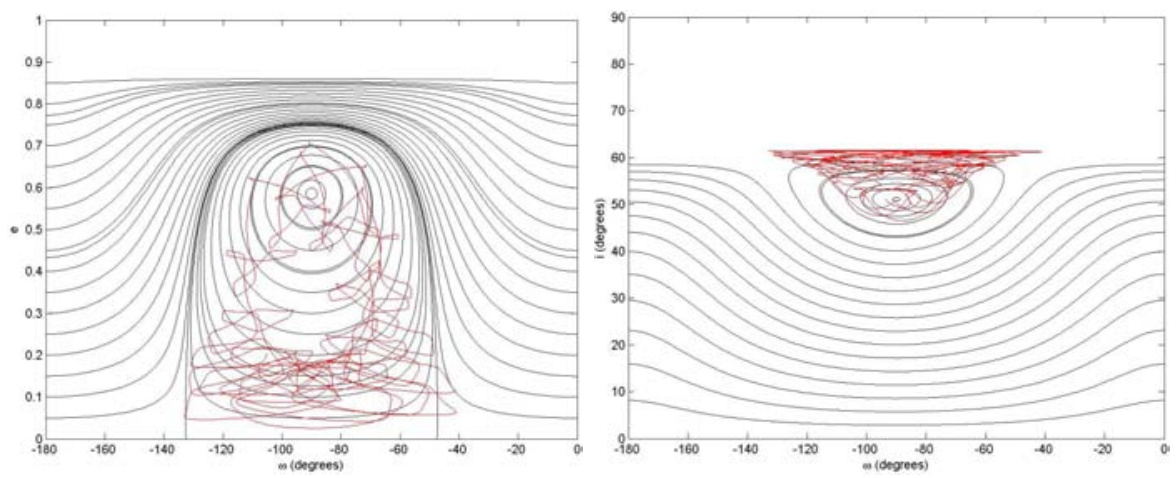

Figure 24. These plots shows eccentricity (left) and inclination (right) as functions of $\omega$. The numerical results shown in red are plotted with contour plots. We can see that the numerical results follow the libration regions, and very low eccentricities are obtained.

We now consider the one-impulse approach for transferring into a circular orbit. This approach involves directly circularizing the orbit from the periapsis passage of the capture trajectory. The cost of this maneuver is $444.5 \mathrm{~m} / \mathrm{s}$ and the resulting orbit has a radius of $1731 \mathrm{~km}$ which corresponds to an altitude of $166 \mathrm{~km}$ above the surface of Europa and an inclination of $51.14^{\circ}$.

All of the transfers to circular frozen orbits computed thus far result in orbits that have different radii 

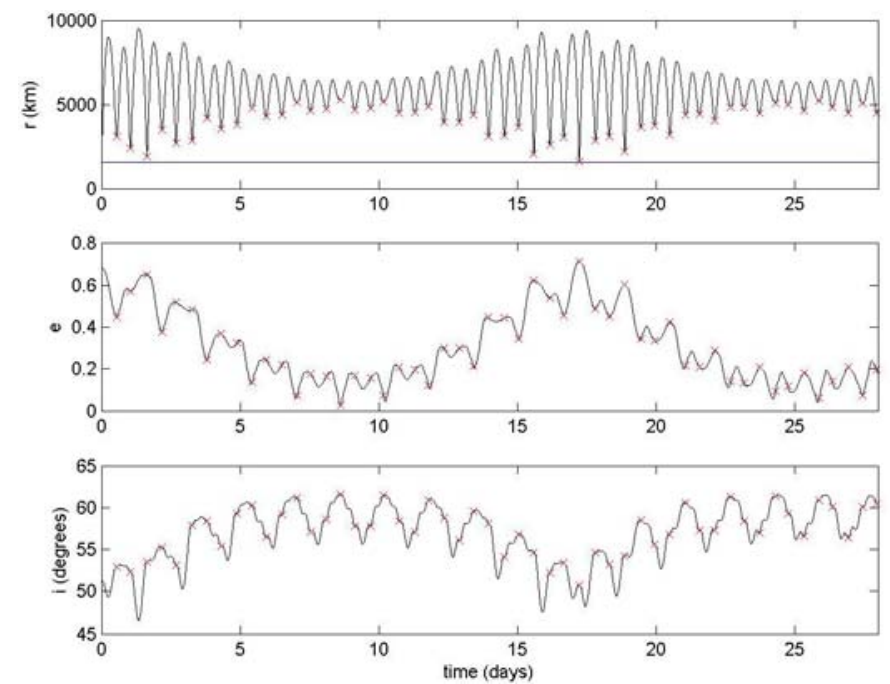

Figure 25. Radius, eccentricity and inclination of the near-frozen elliptic orbit as functions of time. The periapsis passages are indicated by a red ' $x$ ' and the blue line in the top plot represents the surface of Europa.
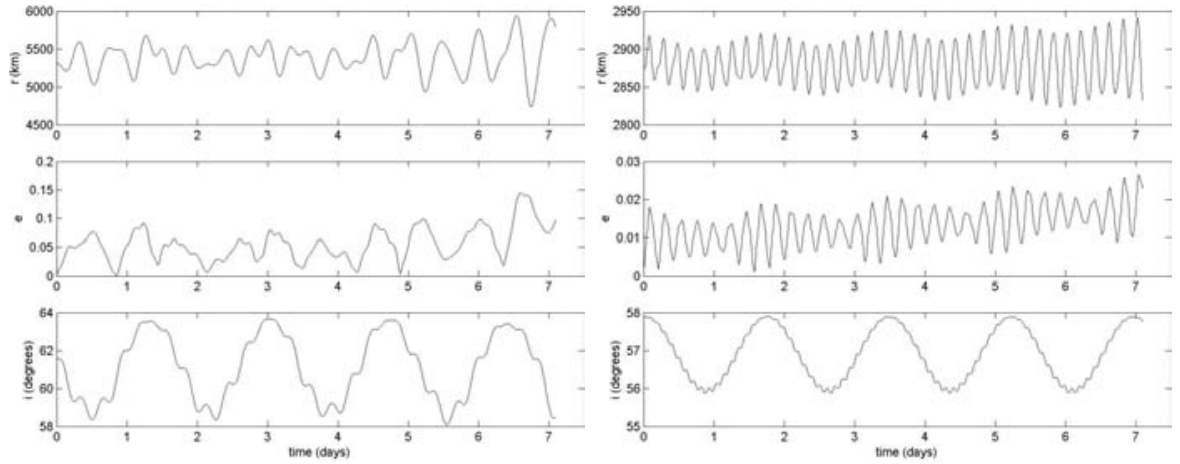

Figure 26. Radius, eccentricity and inclination of both possible circularized orbits over a one week period. The plot on the left corresponds to transferring from the minimum eccentricity of the near-frozen elliptic orbit and the plot on the right corresponds to transferring from a higher eccentricity of that same orbit. We see that in both cases the orbit has a fairly large radius, but is stable for at least one week. 
and inclinations. To compare them, we must consider additional transfers such that we achieve a common orbit for both approaches. We actually consider two common orbits, with inclinations of $61.6^{\circ}$ and $57.9^{\circ}$ and both with a radius of $1731 \mathrm{~km}$. The two inclinations correspond to the inclinations achieved by the two examples of the two-impulse approach and the radius corresponds to the radius achieved by the one-impulse approach. The orbit obtained by the one-impulse approach has an inclination of $51.14^{\circ}$ and so to transfer to inclinations of $61.6^{\circ}$ and $57.9^{\circ}$ costs $247.7 \mathrm{~km} / \mathrm{s}$ and $160.2 \mathrm{~km} / \mathrm{s}$ respectively. The radii of the orbits achieved by the examples for the two-impulse approach are about $5300 \mathrm{~km}$ and $2875 \mathrm{~km}$ and the costs of the Hohmann transfers to reduce those radii to $166 \mathrm{~km}$ are $542.6 \mathrm{~m} / \mathrm{s}$ and $299.7 \mathrm{~m} / \mathrm{s}$ respectively. Table 3 shows a comparison of the costs of the two approaches. We see that the method which uses the dynamics of the system in the vicinity of the elliptic frozen orbits is more efficient, and the difference between the two methods is larger when the final inclination is larger.

\begin{tabular}{lcc}
\hline \hline & \multicolumn{2}{c}{ Method } \\
& Using Dynamics to Circularize & Direct Circularization \\
\hline Example 1 $\left(i=61.6^{\circ}\right)$ & $591.5 \mathrm{~m} / \mathrm{s}$ & $692.2 \mathrm{~m} / \mathrm{s}$ \\
Example 2 $\left(i=57.9^{\circ}\right)$ & $586.6 \mathrm{~m} / \mathrm{s}$ & $604.7 \mathrm{~m} / \mathrm{s}$ \\
\hline \hline
\end{tabular}

Table 3. Comparison of the cost to transfer from the periapsis passage of a capture trajectory to a tightly bound circular orbit. Example 1 corresponds to the circularization taking place at the minimum eccentricity of the near-frozen elliptic orbit and example 2 corresponds to using a higher eccentricity from which to transfer from.

If we compare the total costs of only the two-impulse approach, we see that although the second example is slightly cheaper, an inclination of almost $4^{\circ}$ lower is achieved. If we neglect the Hohmann transfer component of this approach, we see that while the second example achieves a slightly smaller circular frozen orbit, it has a cost of which is 5.5 times greater than the transfer via the first example. Since both circular orbits are stable there is a clear advantage to following the first example. Since the cost to achieve the stable circular orbit is quite small (less than $50 \mathrm{~m} / \mathrm{s}$ ) it is possible that a low-thrust vehicle could achieve this orbit. Following that, the low-thrust vehicle could transition down to a tighter circular orbit with a spiral maneuver.

As another approach to transferring from a safe capture trajectory to a circular frozen orbit, we examine the possibility of performing a simultaneous circularization and plane change maneuver. It is not possible to do this maneuver with our current example, since for this maneuver to be feasible, the argument of periapsis at the periapsis passage must be either $0^{\circ}$ or $180^{\circ}$ and for our current example the argument of periapsis is approximately $-90^{\circ}$. Therefore, we go back to our data of all the periapsis passages of capture trajectories at this energy level, $J=-1.60$ and examine their arguments of periapsis. Figure 27 shows argument of periapsis plotted as a function of eccentricity for all of the periapsis passages of the capture trajectories with $J=-1.60$. In this plot we consider periapsis passages with any radius of periapsis. We see that there are periapsis passages that occur at all possible arguments of periapsis. However, as before, we wish our final orbit to be such that it has a low altitude. Therefore, we restrict the periapsis passages under consideration to those with an altitude less than $250 \mathrm{~km}$. Figure 28 shows the argument of periapsis as a function of eccentricity for the periapsis passages that satisfy this condition. We see that the argument of periapsis only lies between about 50 to $150^{\circ}$ and -150 to $-50^{\circ}$, and so there are no periapsis passages with altitudes close to the surface for which a simultaneous circularization and plane change maneuver can be performed. We can conclude, from comparing Figure 27 and Figure 28, that the periapsis passages with lower arguments of periapsis must have a large radius. We also see that these periapsis passages have lower eccentricities. This is not surprising with the doubly averaged dynamics in mind. In the doubly averaged system, the semi-major axis is constant on average. Therefore, since the capture trajectories originate far from the planetary satellite, for them to have a periapsis passage with a low altitude, the eccentricity must be large. The numerical results support this since when all periapsis passages are considered, they range in eccentricity from $\sim 0.32 \rightarrow 0.75$, but when only those with altitudes less than $250 \mathrm{~km}$ are considered, eccentricity only ranges between about $0.705 \rightarrow 0.75$. Therefore, by limiting the radius of periapsis, we are left with only periapsis passages with large eccentricities. If we consider the averaged dynamics once again, we note that for a trajectory to reach a large eccentricity it must be circulating about a libration region 
and libration regions are centered about $\pm 90^{\circ}$. If we once again examine Figure 28 we see that this is the case. The arguments of periapsis in this plot extend about $\pm 50^{\circ}$ on either sides of $\pm 90^{\circ}$. We can therefore conclude that it is not dynamically possible to obtain a periapsis passage of a capture trajectory that will allow us to perform a simultaneous circularization and plane change maneuver to place the trajectory in a tightly bound circular orbit.

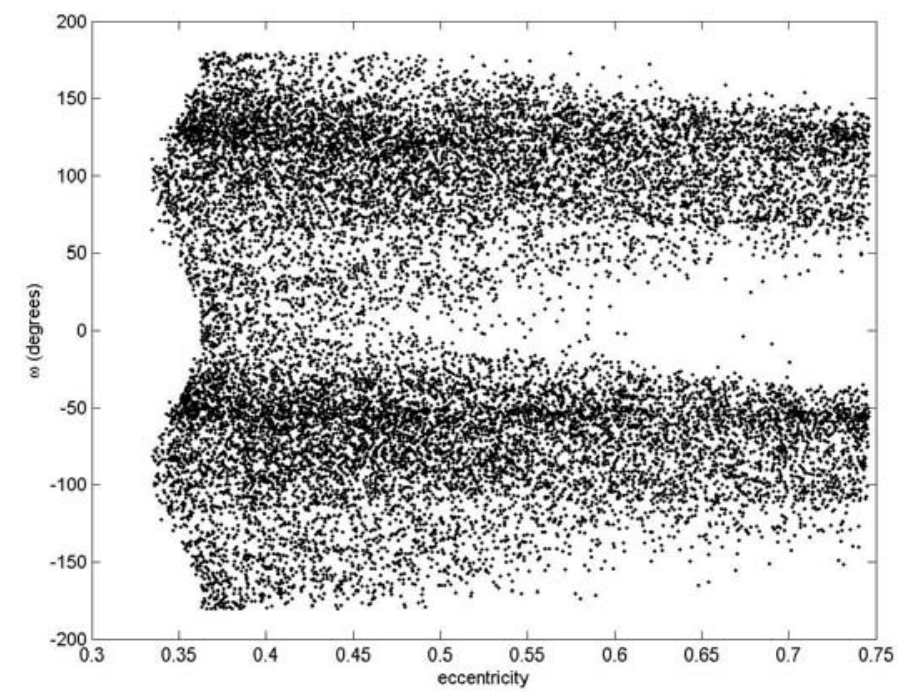

Figure 27. Argument of periapsis as a function of eccentricity for all of the periapsis passages of safe capture trajectories with $J=-1.60$.

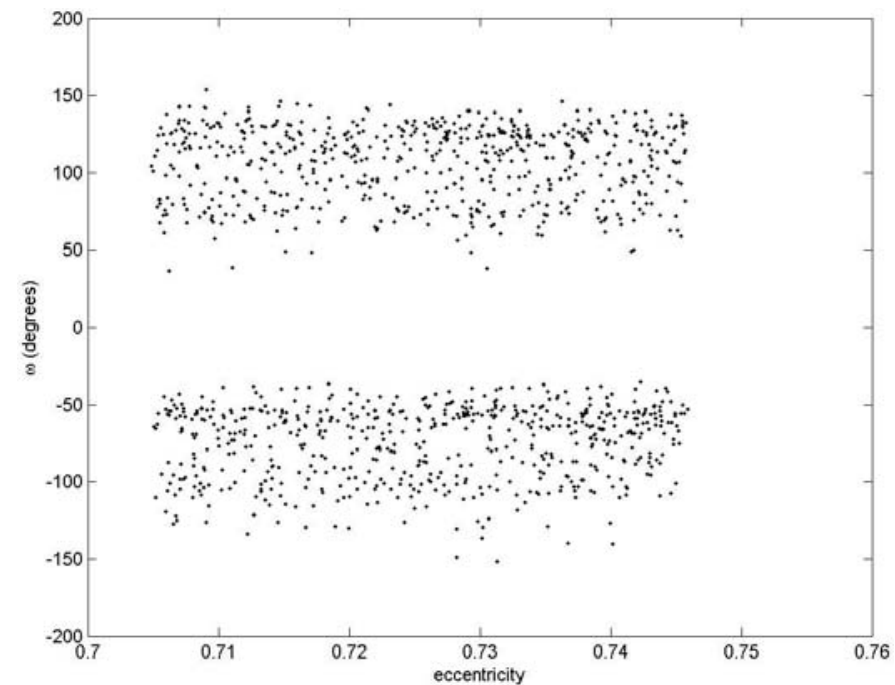

Figure 28. Argument of periapsis as a function of eccentricity for periapsis passages of safe capture trajectories with $J=-1.60$ that have a radius of periapsis less than $250 \mathrm{~km}$ above the surface of Europa.

\section{Conclusion}

We have investigated captured trajectories in the Hill 3-body problem using a Periapsis Poincaré map with application to trajectories about Jupiter's moon Europa. Trajectories that don't impact with Europa or escape for one week time periods have been identified and the regions in which they occur denoted as safe zones. We then evaluated these safe zones to find trajectories for which it is possible to transfer to 
long-term stable orbits. We consider transfers from the periapsis passages of the safe capture trajectories to long-term stable orbits. Orbits considered as possible targets are both elliptic and circular frozen orbits which are either long-term stable or can be stabilized with small control maneuvers. We developed schemes to execute low-cost transfers to both elliptic and circular frozen orbits. Transfers to elliptic frozen orbits can be executed with one burn, while transfers to circular frozen orbits need between 2 and 4 burns. We found that the lowest-cost method to transfer to circular frozen orbits involves using the dynamics of the system to decrease the eccentricity rather than circularizing directly. In particular we describe a low cost sequence that results in a circular orbit that could be reached by a low-thrust spacecraft. From this orbit it could then spiral down into a tighter circular orbit.

\section{Acknowledgments}

M.E. Paskowitz acknowledges the support of the FXB Foundation. This research was supported by a grant from the JPL/Caltech JIMO program.

\section{References}

${ }^{1}$ Broucke, R.A., "Long-Term Third-Body Effects via Double Averaging", Journal of Guidance, Control and Dynamics, Vol. 26, No. 1, 2003, pp.27-32.

${ }^{2}$ Koon, W.S., Lo, M.W., Marsden, J.E. and Ross, S.D., "Heterclinic connections between periodic orbits and resonance transitions and celestial mechanics", Chaos, Vol. 10, No 2., 2000, pp.427-469.

${ }^{3}$ Paskowitz, M.E. and Scheeres, D.J. "Orbit Mechanics about Planetary Satellites", paper presented at the Space Flight Mechanics Meeting, Maui, Hawaii, February 2004.

${ }^{4}$ Scheeres, D.J., Guman, M.D. and Villac, B.F., "Stability Analysis of Planetary Satellite Orbiters: Application to the Europa Orbiter", Journal of Guidance, Control and Dynamics, Vol. 24, No. 4, 2001, pp.778-787.

${ }^{5}$ Villac, B.F., Scheeres, D.J., "Escaping Trajectories in the Hill 3-body Problem and Applications", Journal of Guidance, Control and Dynamics, Vol. 26, No. 2, 2003, pp.224-232.

${ }^{6}$ Villac, B.F., "Dynamics in the Hill Problem with Application to Spacecraft Maneuvers", PhD Thesis, The University of Michigan, 2003. 